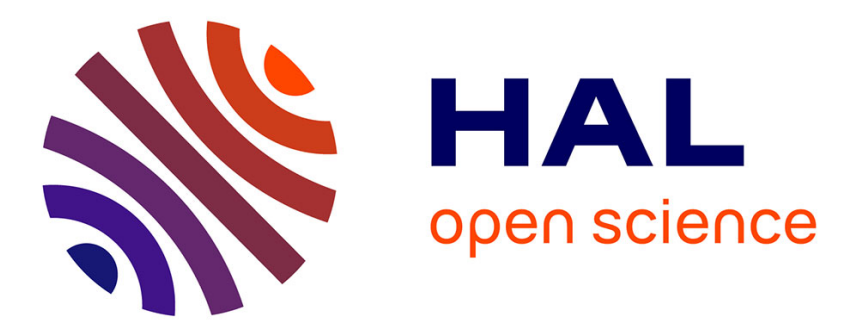

\title{
Position-referenced microscopy: regions of interest localization and subpixel image comparison by means of pseudo-random patterns embedded in cell culture boxes
}

J. Galeano Zea, P. Sandoz, Laurent Robert, E. Gaiffe, J.-L. Prétet, C. Mougin

\section{- To cite this version:}

J. Galeano Zea, P. Sandoz, Laurent Robert, E. Gaiffe, J.-L. Prétet, et al.. Position-referenced microscopy: regions of interest localization and subpixel image comparison by means of pseudo-random patterns embedded in cell culture boxes. European Conferences on Biomedical Optics, Jun 2009, Munich, Germany. pp.110, 10.1117/12.831538 . hal-00472313

\section{HAL Id: hal-00472313 https://hal.science/hal-00472313}

Submitted on 17 Apr 2021

HAL is a multi-disciplinary open access archive for the deposit and dissemination of scientific research documents, whether they are published or not. The documents may come from teaching and research institutions in France or abroad, or from public or private research centers.
L'archive ouverte pluridisciplinaire HAL, est destinée au dépôt et à la diffusion de documents scientifiques de niveau recherche, publiés ou non, émanant des établissements d'enseignement et de recherche français ou étrangers, des laboratoires publics ou privés. 


\title{
Position-referenced microscopy: Regions of interest localization and subpixel image comparison by means of pseudo-random patterns embedded in cell culture boxes
}

\author{
July A. Galeano Zea ${ }^{a}$, Patrick Sandoz ${ }^{a}$, Laurent Robert $^{b}$, Emilie Gaiffe ${ }^{c}$, Jean-Luc Prétet ${ }^{c}$, \\ Christiane Mougin ${ }^{c}$ \\ ${ }^{a}$ Département d'Optique P.M. Duffieux, Institut FEMTO-ST, \\ U.M.R C.N.R.S. 6174 / Université de Franche-Comté, \\ F-25030 Besançon cedex, France \\ ${ }^{b}$ Centrale de technologie MIMENTO, Institut FEMTO-ST, \\ U.M.R C.N.R.S. 6174 / Université de Franche-Comté, \\ F-25044 Besançon cedex, France \\ ${ }^{c}$ Laboratoire de Biologie Cellulaire et Moléculaire, EA3181, IFR133, \\ Université de Franche-Comté, CHU Jean Minjoz, \\ F-25030 Besançon cedex, France
}

\begin{abstract}
This work presents micro-grids integrated to cell culture boxes. These grids allow the systematic registration of the position of a zone observed by optical microscopy in a such way that it is possible to find it again easily for new observations for instance after culture on drug injection. The position knowledge allows also the numerical superimposition of recorded images in a common position reference system with a sub-pixel precision. It become thus straightforward to perform a site by site analysis of the possible evolutions that may have occurred in the biological medium.
\end{abstract}

Keywords: optical microscopy, region of interest localization, space-frequency analysis, pseudo-periodic pattern, linear shift register sequences, phase measurements, biological application

\section{INTRODUCTION AND PRINCIPLE}

Beyond sharp visualization of media, biology research is now requiring microscopy systems able to trace dynamic phenomena occurring in living systems. For short constant of time, several approaches were proposed for multi-dimensional imaging, as for instance fluorescence life time imaging (FLIM) or fluorescence resonance energy transfer (FRET). ${ }^{1}$ The case of long time constants may seem simpler since a long-term microscopy observation is sufficient as performed by videomicroscopy. However, the mobilization of an entire microscopy unit for the observation of a single field of view evolving slowly is not optimum, especially if cell culture conditions require a controlled environment to be maintained. Position-referenced microscopy proposed here is a solution to combine long-term observation of well identified regions of interest with cell culture boxes transfers from culture environment to microscopy units. Cell culture boxes are taken out of their living environment for the short while necessary for observation and then return to culture. Several regions of interest can thus be observed in parallel and the microscope unit remains available for other uses. This way of operation is made possible by inserting a position reference pattern within the cell culture box structure. This pattern allows the absolute position and orientation of the imaged field to be determined in order to be localized easily at the time of next observations. Furthermore digital image superimposition is performed with a subpixel accuracy and allows the accurate quantification of biological media transformations.

Further author information:

E-mail: july.galeano_zea@univ-fcomte.fr

patrick.sandoz@univ-fcomte.fr 

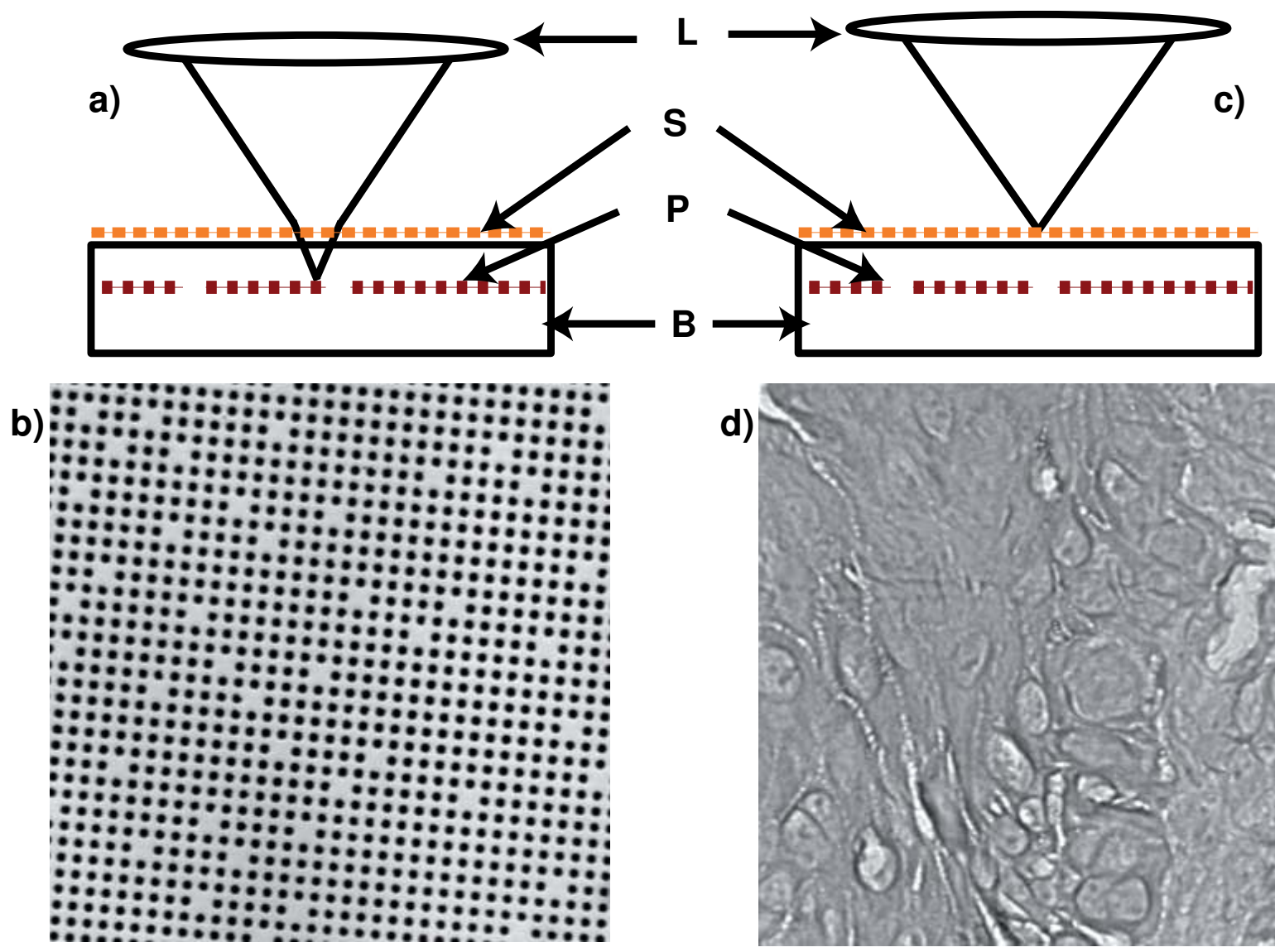

d)

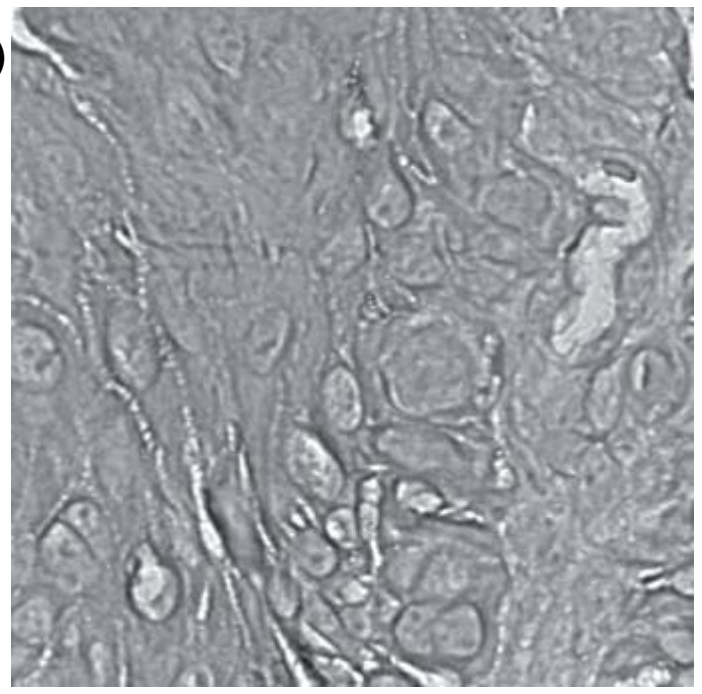

Figure 1. L: lens; S: biological specimen; P: embedded position reference pattern; B: culture box bottom; a) focus on the embedded position reference pattern; b) image of the local area of the position reference pattern; c) focus on the specimen; d) usual image of the specimen.

\section{STATE OF THE ART AND CONCEPT}

Several position reference systems were proposed on microscope slides for the coarse localization of regions of interest. The concepts reported integrate specific features on the slides that are geometrical and/or alphanumerical. ${ }^{2-6}$ The latter subdivide the slide area in a set of smaller areas that allow a visual identification of zone observed. Those methods propose some basic help to the microscope user for a coarse localization on the microscope slide. Position-referenced microscopy proposed here presents additional functionalities with respect to these coarse localization techniques. The basic idea is to use the short depth of focus of microscope objectives for the insertion of a reference position pattern in the depth of the specimen support as depicted in Fig. 1. Two images with the same lateral coordinates are observed and registered successively by a simple 'Z' adjustment of the focus depth. These images can be recorded without crosstalk provided that the depth of the reference pattern relatively to the specimen plane is larger than the depth of focus of the objective. This principle of operation associates a local image of a reference position pattern with the area of the specimen under observation. The former image is used for position computation that describes the accurate localization of the region observed with respect to the specimen support. Once a region of interest has been identified by means of the corresponding image of the position reference pattern, it becomes easy to find the same area at the time of next observations. Furthermore, a set of images of a same zone recorded at different time can be adjusted numerically to be displayed in a common pixel reference frame in order to obtain high accurate image superimposition. This capability allows quantitative evaluation of the specimen modifications with a subpixel resolution. 

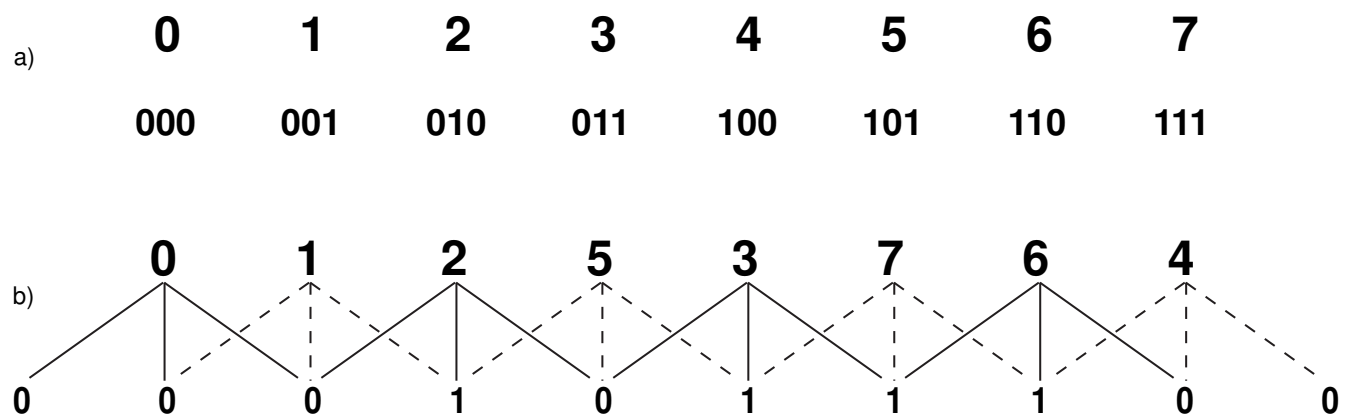

Figure 2. Example of imbricated binary sequence with words of $\mathrm{N}=3$ bits: a) Usual sequence, each word requires three bits to be coded; b) Example of a LFSR sequence, each word shares N-1 bits with its neighbors and thus the bit number necessary for the whole sequence tends to be equal to the number of words.

\section{POSITION REFERENCE PATTERN DESIGN, PROCESSING AND RESULTS}

Fig. 1.b gives an idea of the position reference pattern design. The position reconstruction is based on a combination of complementary fine and coarse position detection schemes. The fine detection - able to furnish a high level of interpolation between pixels - is based on the digital processing of a periodic grid. The periodic distribution of points corresponds to well defined spatial frequencies that can be extracted from each other in the Fourier domain after Fourier transform. ${ }^{7,8}$ Each of them is associated to a linear variation of the corresponding spatial phase in the image domain that can be reconstructed after inverse Fourier transform. By approximating this spatial phase plane by least square fitting we are sensitive to subpixel displacements. A resolution better than $10^{-2}$ pixel was demonstrated from digital images coded on 256 gray levels. Because of the pattern periodicity, this phase analysis suffers from $2 \pi$ phase ambiguities due to the unknown order of the lines and columns of the periodic pattern. Therefore at this stage, the position is reconstructed with an uncertainty of an entire number of pattern periods. The removal of these ambiguities is the aim of the coarse position detection scheme. The latter is based on periodicity alterations that appear as missing points in figure 1.b. These missing points make the pattern pseudo-periodic by distributing in an unique way the missing points over the whole two-dimensional encoded area. This encoding design is obtained by extending to two dimensions binary sequences resulting from linear feedback shift registers (LFSR). ${ }^{9}$ The basic principle consists in encoding a sequence of $\left(2^{N}\right)$ positions by using a shift register of length N. With appropriate linear feedback of the shift register with XOR gates, the words produced successively by the $\mathrm{N}$ register values describe all possibilities, i.e. $2^{N}-1$ different words (only the zero value is missing and can be introduced in case of computer generated sequences). The effect of this encryption method is depicted in Figure 2 for the case of words of three bits. Because of the shift register principle, successive words share necessarily $\mathrm{N}-1$ bits with immediate neighbors. It is thus possible to retrieve the word value by combining $\mathrm{N}$ consecutive bits centered on the current position. By using this technique, the encoding of a sequence of $2^{N}$ words requires only $2^{N}+N-1$ bits. The only requirement is the knowledge of a look-up table the conversion of the words read into their actual position along the sequence. The extension of this principle to two dimensions is obtained by reproducing identical sequences among which some are shifted with respect to other ones. ${ }^{10}$

The reconstruction of the actual position of the zones observed assumes the correct decoding of the missing point distribution. Specific algorithms and software were developed successfully for this purpose. The orientation of the pseudo-periodic pattern with respect to the pixel frame is also retrieved from the spatial phase plane approximation. Therefore we obtain an unique and absolute position definition of any observed area with respect to the whole pseudo-periodic pattern. Since the position reference pattern is embedded within the culture box structure, we are not affected by the positioning of the specimen on the microscope stage and we are able to come back to an area observed earlier in order to observe potential modifications. Fig. 3.a presents an example in which the visibility of the missing point distribution is low because of shadow effects and illumination non uniformity. In fact during image processing, the spatial phase data is used for the determination of a local 

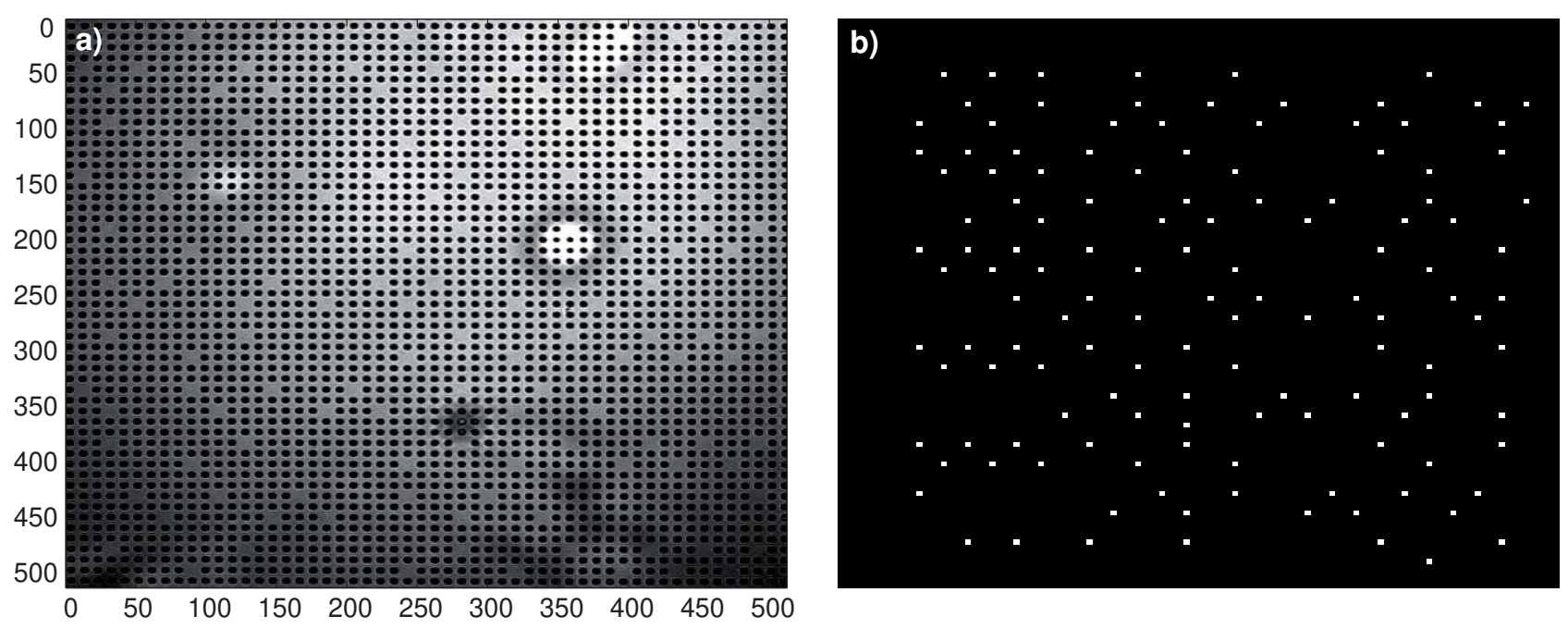

Figure 3. a) Example of the recorded image of the position reference pattern in presence of shadows and non uniform illumination; b) Correct distribution of missing points as reconstructed from Fig. 2.a.

visibility coefficient suitable for distinguishing between low intensity levels. As can be seen in Figure 2.b the algorithms developed are efficient enough to identify properly the missing point locations and to retrieve the actual position of the observed area. Fig. 4a illustrates the digital superimposition of images recorded at different times. To obtain an easy visualization of the superimposition, two black and white images of approximately the same tissue area were put respectively in the red and green channels of an RGB color image after coordinate adjustment in a common position reference system. The yellow shade results from red and green mixing with equal intensities and its quality demonstrates the sharpness obtained in image superimposition. In case of object modification between image recordings, the red and green levels mismatch resulting in red and green spots in the respective object positions. The accuracy achieved in position determination and image superimposition is equal to a fraction of pixel. In a general case, the residual error remaining on position, or image superimposition, is about 10 times smaller than the diffraction limit of the lens used. This level of performance makes these residual errors negligible compared to the image definition. Fig. 4.b shows a cell culture box in which the central zone has been encoded with a position reference pattern by photolithography. At present time, our feasibility tests concern only a few tens square millimeters but can be extended to several square centimeters by adapting the binary sequences. We also work on coverslips that can be glued to culture boxes when their thickness is too large compared to the working distance of microscope objectives.

The position of the fields of view observed is monitored by means of a user interface developed specifically with Matlab. The latter is shown in Fig. 5. If gives the possibility to chose the image used as reference and to locate the current image with respect to the reference one. It is possible to represent both images in a common position reference system and thus to determine the motion to apply to the microscope stage in order to come back on the given region of interest. The motion can be operated either manually or with a motorized stage. Once a sufficient superimposition of the fields of view is achieved, numerical image superimposition is proposed that allows a sharp analysis of the possible evolution that may have occurred i the biological preparation during the time lapse separating these observations.

\section{CONCLUSION}

The principle and demonstration of position referenced microscopy are given. Microscopic pseudo-periodic patterns have been designed and manufactured on both microscope slides, coverslips and cell culture boxes. The technique allows finding easily regions of interest observed previously as well as the accurate superimposition of images of a same zone recorded at different times. Image processing software as well as user interfaces were 

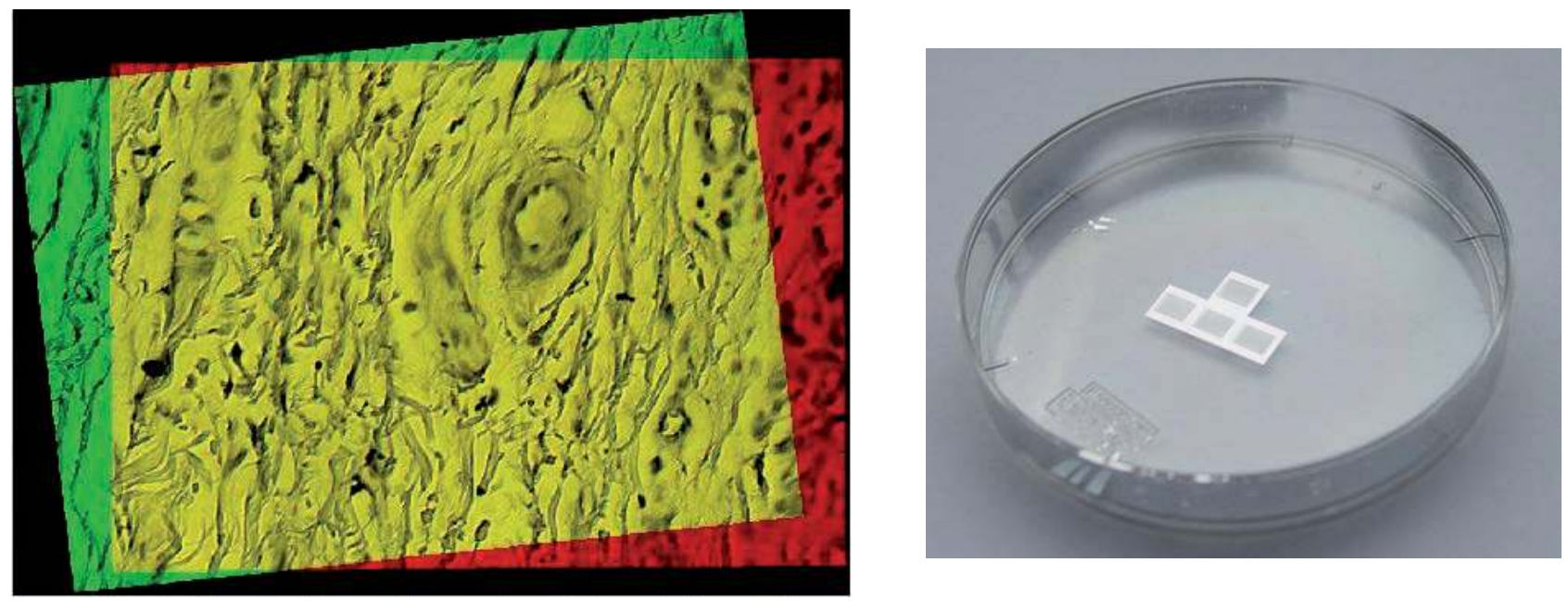

Figure 4. a) Example of digital superimposition of images recorded at different times; red: first image; green: second image. The yellow shade results from superimposition of red and green with equal level. b) Image of an encoded zone within a 3" plastic culture box. Experiments were carried out with encoded surfaces of $3 \times 3.6 \mathrm{~mm}^{2}$.

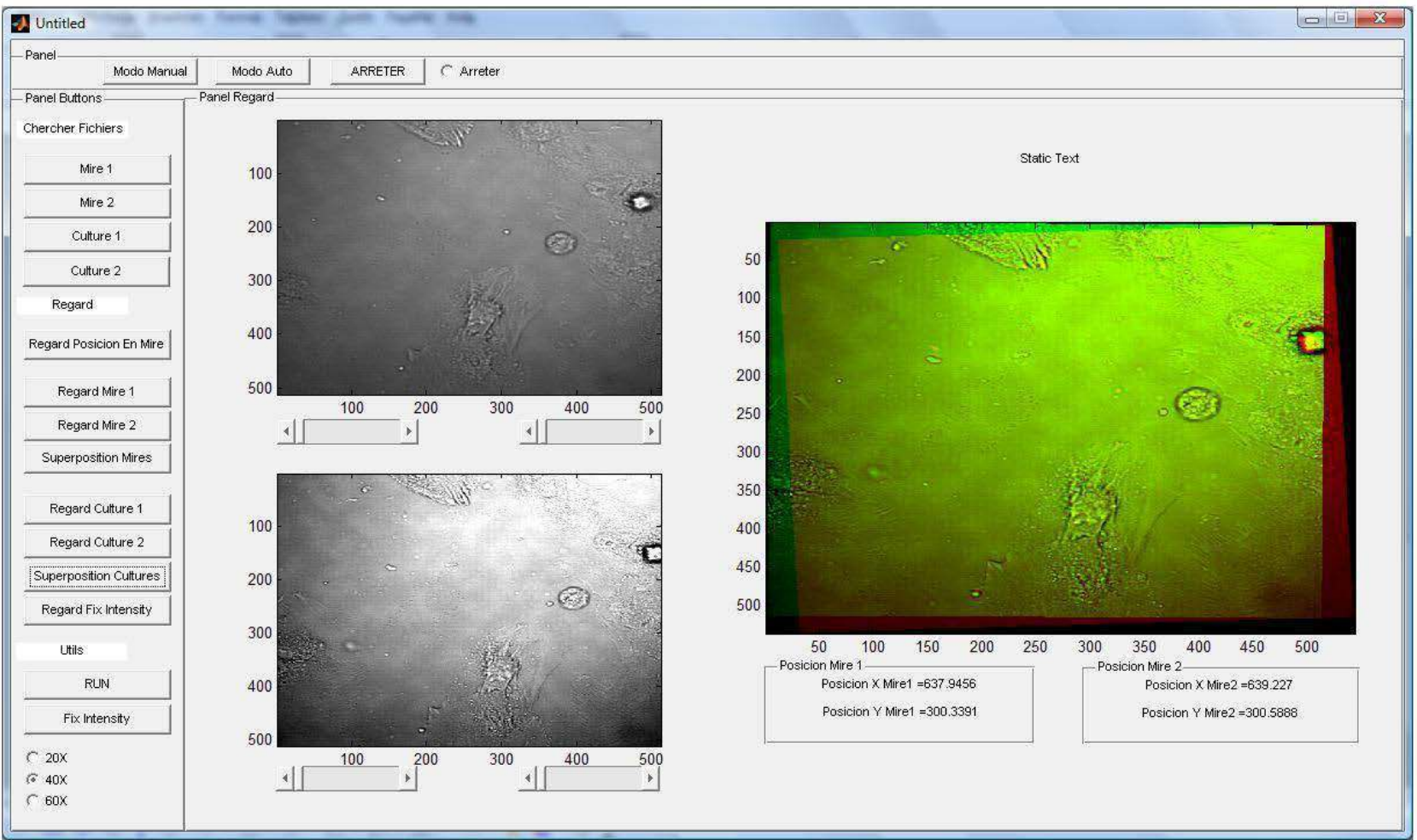

Figure 5. User interface developed with Matlab. It allows the monitoring of recorded images and the superimposition of chosen images in an unique pixel frame. It can be use to determine the distance of the current view from a region of interest as well as to compare images of a single zone observed at different instants of time. 
developed specifically and make the technique available for use in actual microscopy experiments. This method is being applied to biological research, especially to the study of apoptotic bodies issued from cancer cells.

The use of pseudo-periodic pattern for position measurements has also been used for metrology purpose, for instance for vibration quantification. ${ }^{11,12}$ It has also been used with an interference objective for the following of position along the six degrees of freedom. ${ }^{13}$

\section{ACKNOWLEDGMENTS}

We would like to acknowledge Région de Franche-Comté for project funding (UFC 39SB) and Institut National du Cancer (INCa) for supporting PhD work of July Andrea Galeano Zea.

\section{REFERENCES}

1. Bastiaens, P. and Squire, A., "Fluorescence lifetime imaging microscopy: spatial resolution of biochemical processes in the cell," Trends in Cell Biology 9(2), 48-52 (1999).

2. Feldman, B., "Microscope slide," (Jan. 15 1980). US Patent 4,183,614.

3. Ruddle, F. and Lin, P., "Method for engraving a grid pattern on microscope slides and slips," (Nov. 15 1983). US Patent 4,415,405.

4. Lin, P. and Ruddle, F., "Photoengraving of coverslips and slides to facilitate monitoring of micromanipulated cells or chromosome spreads.," Exp Cell Res 134(2), 485-8 (1981).

5. Dimou, G. and Pang, T., "Process for manufacturing a cover glass with a viewing field," (June 16 1998). US Patent 5,766,677.

6. Hause, L. and Jeutter, D., "Mapping method for a microscope slide," (July 28 1998). US Patent 5,786,130.

7. Sandoz, P., Ravassard, J., Dembelé, S., and Janex, A., "Phase-sensitive vision technique for high accuracy positionmeasurement of moving targets," IEEE Transactions on Instrumentation and Measurement 49(4), 867-872 (2000).

8. Sandoz, P., Bonnans, V., and Gharbi, T., "High-accuracy position and orientation measurement of extended two-dimensional surfaces by a phase-sensitive vision method," Applied optics 41(26), 5503-5511 (2002).

9. Golomb, S., [Shift register sequences], Aegean Park Press Laguna Hills, CA, USA (1981).

10. Sandoz, P., Zeggari, R., Froehly, L., Pretet, J., and Mougin, C., "Position referencing in optical microscopy thanks to sample holders with out-of-focus encoded patterns," Journal of Microscopy 225(3), 292-302 (2007).

11. Sandoz, P., Friedt, J., and Carry, E., "In-plane rigid-body vibration mode characterization with a nanometer resolution by stroboscopic imaging of a microstructured pattern," Review of Scientific Instruments $\mathbf{7 8}$, 023706 (2007).

12. Sandoz, P., Friedt, J., and Carry, É., "Vibration amplitude of a tip-loaded quartz tuning fork during shear force microscopy scanning," Review of Scientific Instruments 79, 086102 (2008).

13. Sandoz, P., "Nanometric position and displacement measurement of the six degrees of freedom by means of a patterned surface element," Applied Optics 44(8), 1449-1453 (2005). 